\title{
Meta
}

Journal des traducteurs

Translators' Journal

AWAISS, Henri et HARDANE, Jarjoura, dir. (2010) : Jean-René

Ladmiral, le dernier des archéotraductosaures, interviewé par l'ETIB, Beyrouth, École des traducteurs et d'interprètes de Beyrouth, Faculté des Lettres et des Sciences Humaines, Université Saint-Joseph, Collection « Sources-Cibles », 122 p.

\section{Muguras Constantinescu}

Volume 57, numéro 4, décembre 2012

URI : https://id.erudit.org/iderudit/1021238ar

DOI : https://doi.org/10.7202/1021238ar

Aller au sommaire du numéro

Éditeur(s)

Les Presses de l’Université de Montréal

ISSN

0026-0452 (imprimé)

1492-1421 (numérique)

Découvrir la revue

Citer ce compte rendu

Constantinescu, M. (2012). Compte rendu de [AwaIss, Henri et HARDANE, Jarjoura, dir. (2010) : Jean-René Ladmiral, le dernier des archéotraductosaures, interviewé par l'ETIB, Beyrouth, École des traducteurs et d'interprètes de Beyrouth, Faculté des Lettres et des Sciences Humaines, Université Saint-Joseph, Collection « Sources-Cibles », 122 p.] Meta, 57(4), 1090-1092. https://doi.org/10.7202/1021238ar d'utilisation que vous pouvez consulter en ligne. 
of read to be truly funny. In practical terms, it means that she dealt with the colloquial style of the original by playing with the structure of her text, sometimes separating paragraph-long sentences and adding many conjunctions, commas and dashes. Maher also explains how she preserved the cultural identity of the novel by keeping Italian words, and by exploiting the target readers' background knowledge of Italy, in order to "increase their appreciation of [the text's] humorous and ironic potential" (p. 144). She concludes the chapter with observations about the translation process and the overall reception and marketing efforts of Mal de pietre in the target culture. Finally, the last chapter is a short reflection on the creativity needed to translate humorous texts of all kinds, as demonstrated by the previous case studies. Maher states that translation can be the site of ingenious gain and that it can ultimately open up "the target literary system to new cultures and influences" (p. 162). She recalls how every minor decision can affect the whole translation, and the importance of both micro and macro aspects in the interpretation of a text, humorous or not. She also notes that if the translators sometimes seem to favor more conservative translations over recreation, it is because he risks having all the blame and none of the credit. Recreation and Style ends with further research ideas inspired by the conclusion of each case study and that could deepen our knowledge of the interaction between humour, creativity, culture and translation.

In the context of translation studies, the originality of Maher's work is that it comprehends humour as a very broad and multifaceted phenomenon. Instead of focusing on specific comic elements such as puns or one-liners, she studies other kinds of humour that were seldom explored in translation studies, from grotesque to cross-referential parodies and subtle innuendos. Besides, Maher completely understands the crucial importance of cultural backgrounds in comedy, as she includes elements such as critical reviews, book covers, marketing initiatives and previous translations in her reflection. Even if these paratextual considerations often go beyond the translator's role or power, they will have a major impact on the reception of his or her work in the target culture. The practical recommendations and suggested alternative translations found throughout the book are also a welcomed addition. Recreation and Style is not only a relevant and original contribution to the study of humour in translation, but also a heartfelt tribute to literary translators and their underestimated creative abilities.

Hugo Vandal-Sirois Université de Montréal, Montréal, Canada

\section{REFERENCES}

Diot, Roland (1989): Humor for Intellectuals: Can it Be Exported and Translated? The Case of Gary Trudeau's In Search of Reagan's Brain. Meta. 34(1):84-87.

VANDAELE, Jeroen (2010): Humor in translation. In: Yves GAMBIER, ed. Handbook of Translation Studies. Vol. 1. Amsterdam/Philadelphia: John Benjamins Publishing Company, 147-152.

Awaiss, Henri et Hardane, Jarjoura, dir. (2010): Jean-René Ladmiral, le dernier des archéotraductosaures, interviewé par l'ETIB, Beyrouth, École des traducteurs et d'interprètes de Beyrouth, Faculté des Lettres et des Sciences Humaines, Université Saint-Joseph, Collection «SourcesCibles», $122 \mathrm{p}$.

Paru en 2010, «année Ladmiral» par la multitude d'événements organisés un peu partout autour de l'œuvre et de la personnalité du grand traductologue, l'ouvrage publié à l'Université Saint-Joseph de Beyrouth dans la collection "Sources-Cibles", dirigée par Henri Awaiss et Jarjoura Hardane, innove une formule originale; il réunit, dans une première partie, les questions des chercheurs, cadres et étudiants, et les réponses du grand chercheur, en la mémorable journée-hommage du 22 avril, placée sous le signe de la créativité. La deuxième partie présente succinctement l'œuvre de Jean-René Ladmiral.

Ce livre collectif commence par un «Prélude» sous la forme d'un conte où, bon esprit, Henri Awaiss raconte à Saint-Exupéry qu'Il est revenu, habillé d'une laine blanche et portant un foulard bleu, pour visiter la huitième planète, celle de la traductologie. Lui, ce Grand Petit Prince est, bien sûr, Jean-René Ladmiral qui, avec plaisir, fraîcheur et une grande disponibilité, se prête à ce jeu de questions-réponses. Le rôle d'animatrice est revenu à Elsa Charabati, qui a su bien gérer le temps d'une journée et d'une rencontre exceptionnelles.

La lecture de cet ouvrage éclairant autant qu'agréable par sa formule, nous fait découvrir et redécouvrir le chercheur aux solides repères théoriques mais également celui qui tâtonne, nuance, raffine encore ses idées ou en découvre, avec gravité ou enjouement, de nouvelles, grâce à cet entretien pluriel. Elle nous fait découvrir aussi l'essentiel de la traductologie ladmiralienne, en formule décontractée, métaphorique, «exotérique et accrocheuse» (p. 60), qui laisse pourtant entrevoir aux connaisseurs son fertile noyau scientifique.

Le volume respecte le déroulement de la journée-hommage où les interviewers ont posé 
leurs questions sous forme thématique et se sont succédé en ordre alphabétique; de ces nombreuses et stimulantes questions, nous n'en retiendrons que quelques-unes. Ainsi, à la question de May Akl sur le statut de la traductologie et de sa scientificité, la réponse de Ladmiral est celle, déjà formulée plusieurs fois dans ses écrits, du praticien de la traduction qui repousse l'idée d'une seule, bonne et vraie théorie, au nom d'un bénéfique pluralisme théorique. D'ailleurs, selon lui, ce pluralisme et l'apparent déficit de scientificité sont spécifiques aux sciences de l'homme en général et, dans le cas de la traductologie, les choses se passent au grand profit «d'une exploration plus fine, plus détaillée de ce phénomène complexe de la traduction» (p. 17)

A l'étonnement de Henri Awaiss devant la page 112 des Théorèmes, où son invité affirme que le traducteur est un co-auteur et un ré-écrivain et non pas un auteur et un écrivain tout court, comme on le croit à l'ETIB, répond l'étonnement de Ladmiral qui voit dans tout ré-écrivain un écrivain mais qui travaille sous la pression de l'original; d'où une "écriture exigeante» et "de précision» (p. 21).

Plus tard, au cours de la journée et de l'entretien, le traductologue invité est revenu sur cette idée pour la nuancer et dire que le traducteur est un «ré-écrivain complètement» qui produit un «texte à part entière», qui fait un travail «créatif, inventif, littéraire» (p. 74) mais non pas un auteur car il n'a pas l'autorité du texte et il n'est pas à l'origine du texte traduit qui «s'autorise de ce qui le précède» (p. 75). On retiendra la nuance...

La question de Gina Saad sur le salto mortale de la traduction, situé entre la déverbalisation et la reformulation, et son éventuel remède au moyen de l'herméneutique est l'occasion pour l'interviewé de revenir sur la part de mystère qui enveloppe ce moment du traduire qui consiste, semble-t-il, en ses termes enjoués, à "s'arracher à la fascination" (p. 49) du texte, tout en mobilisant sa "puissance d'expression» (p. 50) pour produire le rendu; le conseil du praticien et du professeur pour sortir de la fascination et, en fait, de la difficulté, est bien simple: s'imaginer qu'on raconte le texte à son mari ou à sa grand-mère (qui ne travaillent pas dans le domaine); cet entourage familier et non contraignant aide à débloquer la situation et à produire la formulation attendue. Compte tenu des autres réponses du traductologue, on reconstitue les circonstances les plus favorables de cette production où l'herméneutique et la culture générale ont leur poids, sans annuler pour autant la part de mystère.

La place de la culture générale dans la formation du traducteur préoccupe sans doute le grand traductologue car il y revient plusieurs fois, indigné contre ceux qui y voient une simple somme de connaissances, pour en proposer une définition plus souple et, en même temps, plus complexe: «Or la culture générale c'est plutôt la capacité intellectuelle, psychologique à maîtriser des situations imprévues à la lumière de certaines expériences de lecture, dont la culture générale a été l'occasion. » (p. 32)

Dans l'entourage de la culture générale et de la culture traductologique et, en quelque sorte, à sa source, se trouve l'indispensable travail de documentation en dehors duquel la traduction est difficile, voire impossible, d'où l'exhortation du chercheur, adressée aux traducteurs en herbe, d'aller consulter les livres, les encyclopédies, l'Internet, les films, les sources.

La question de May El Haddad sur l'histoire de la traduction a donné l'occasion de dévoiler le visage grave du traductologue qui considère que l'histoire de la traduction est une «entrée en traductologie en pente douce» (p. 34), qu'elle constitue une bonne préparation à la réflexion traductologique et, surtout, qu'elle amène à «relativiser les problèmes, à désabsolutiser, à relativiser. » (p. 35)

L’opposition entre «l'aristocratie des théoriciens traductologues» et "le prolétariat des praticiens traducteurs» (p. 37), plus visible encore par l'explosion du discours théorique sur la traduction, objet d'une autre question, attire une réponse de bon sens qui souligne la complexité de la traduction et l'importance de la pratique: «Comme la traduction est un phénomène beaucoup plus complexe qu'on ne pense, c'est à partir d'une expérience qu'on en a, qu'on est sans doute en position d'en faire une théorie plus efficace.» (p. 38)

Si parfois les questions des étudiants ont eu un grain de désinvolture ou d'ingénuité, elles se sont avérées stimulantes pour Jean-René Ladmiral qui s'est mis à leur écoute, en entrant dans le jeu des métaphores proposées par les jeunes «étibiens". À la question de l'étudiante Carole Berbari de savoir si Ladmiral, qui prône quelque part un œcuménisme traductologique, se voit comme un «pape» de la traductologie, en toute courtoisie, l'interviewé cède cet honneur à André Clas et garde pour lui seule la fonction de cardinal. En échange, il soutient avec gravité la cause d'un rapprochement, d'un effort de collaboration des différentes «confessions » théoriques pour faire apparaître et reconnaître les convergences, tout en gardant les divergences et les identités, dans le but de centrer l'attention sur la complexité de la pratique.

À la question de l'étudiante Béatrice Tohmé sur la traduction de la culture, Ladmiral évoque dans sa réponse les solutions de la sur-traduction et de la sous-traduction, de l'idiomatisation et de la terminologisation, qui peuvent être adéquates, en fonction du texte à rendre, et souligne le fait qu'il s'agit d'abord de comprendre la culture d'origine 
qui sous-tend le texte pour en trouver ensuite de bons équivalents.

Dans cet entretien pluriel les questions et les réponses ont touché également à d'autres problèmes préoccupants autant pour les traducteurs, les interprètes et leurs formateurs que pour les traductologues: les qualités d'un texte traduit, la créativité du traducteur et ses limites, le bilinguisme, les activités complémentaires à la traduction, la traduction en tant qu'atelier d'écriture, l'intertextualité, les étapes de l'interprétation, la traduction comme communication interculturelle, etc.

Malgré son prestige et son piédestal, l'interviewé s'est permis de se montrer parfois, en toute franchise, désemparé devant quelques questions comme celle sur la qualité de la langue maternelle ou, changeant de rôle, de poser lui-même des questions difficiles comme: «Le bilingue a-t-il une langue maternelle?» (p. 66)

En jouant sur le paradigme métaphorique et stimulé par l'ambiance d'une oralité conviviale, l'invité a donné des formulations mémorables: «en dernier recours, en traduction et en apprentissage, c'est la pratique qui fait l'arbitrage» (p. 27), la traduction est une "ascèse exigeante et productive» (p. 3), «le fantasme de la traduction automatique» (p. 30), «l'interprétation c'est en quelque sorte le point d'incandescence de la traduction » (p. 91) mais aussi sur soi-même, en se définissant comme «le dernier des archéotraductosaures de l'ère précourielique tardive» (p. 71), d'où le titre de l'ouvrage.

La dernière partie, comprenant une «Notice biographique» et "Publications», donne une image de l'ampleur des activités et de la recherche de JeanRené Ladmiral; aux nombreux titres bien connus s'ajoutent aussi des recueils à paraître comme: Éléments de traductologie: Recherches et applications et Pour une philosophie de la traduction.

Voilà donc l'ouvrage collectif de l'ETIB sur l'auteur des Théorèmes sur la traduction, riche et stimulant, et particulièrement agréable et attrayant par sa forme d'entretien qui dévoile un visage moins connu - réflexif et ludique, grave et gracieux - de Jean-René Ladmiral.

Muguras Constantinescu Université Stefan cel Mare, Suceava, Roumanie

\section{RÉFÉRENCES}

LAdMiRAL, Jean-René (1994): Traduire: théorèmes pour la traduction. Paris: Gallimard.

Contribution réalisée dans le cadre du programme CNCS PN-II-ID-PCE-2011-3-0812 (Projet de recherche exploratoire) Traduction culturelle et littérature(s) francophone(s): histoire, réception et critique des traductions, Contrat 133/2011. 